\title{
Prevalence of Plasmodium Falciparum among Nigerians in Abuja and Central States: A Comparative Analysis of Sensitivity and Specificity Using Rapid Diagnostic Test and Microscopy as Tools in Management of Malaria
}

\section{Peter Asaga Mac ${ }^{1,2^{*}}$, Hussaini Fatima Asheadzi², Amuga Gideon ${ }^{2}$, Prerna Thaker ${ }^{3}$ and Philomena Airiohuodion $^{4}$}

\author{
${ }^{1}$ Albert Ludwig University Freiburg, Institute of Human Virology, Germany \\ ${ }^{2}$ Nasarawa State University, Keffi, Nigeria \\ ${ }^{3}$ University of Freiburg, Centre for Medicine and Society, Germany \\ ${ }^{4}$ Zankli Medical Centre, Abuja, Nigeria
}

*Corresponding author: Peter Asaga Mac, MD/PhD, Institute of Human Virology, University of Freiburg, Hermann-Herder-Str. 11• 79104, Freiburg, Germany

\section{Abstract}

Introduction: In Nigeria, malaria is endemic with comorbidities such as dengue, chikungunya, Zika, Tuberculosis and HIVIAIDS which makes malaria a deadly infection. Malaria accounts for $25 \%$ of infant Mortality and $30 \%$ of childhood mortality [1]. The use of laboratory methods has become necessary because health workers cannot identify malaria cases reliably using clinical signs and symptoms alone [2,3].

Methods: A cross-sectional study of 403 consenting healthy individuals who came to National hospital, Abuja and Nasarawa State University hospital, Keffi for medical examination were recruited from April 2017 through July 2017. The socio-demographic charactersitics of each participant was obtained through oral consultation. Information collected included the sex, age, occupation and locality to which they belonged. The gold standard tool (microscopy) and RDTs were used to determine the prevalence of malaria infection, the Sensitivity and specificity of RDT was compared to Microscopy gold standard.

Results: The prevalence of malaria infection among the 403 subjects was $54.4 \%$ and $44.9 \%$. There was a significant difference between the two methods employed $\left(X^{2}=52.171\right.$, $p<0.001)$. The preponderance of malaria in relation to age was highest $(78.9 \% ; 4.7 \%)$ for ages (above 51 years), those under 20 years demonstrated a pervasiveness of $(57.4 \%$; $31.9 \%)$ through the two techniques. A prevalence of $(64 \%$; $20 \%$ ) was observed in the age group (41-50 years) and to a
\end{abstract}

lesser extend $(54.3 \% ; 28.6 \%)$ those years $31-40$ years and $(52.3 \% ; 43.7 \%)$ age $21-30$ years. There were no significant differences between all age groups $\left(X^{2}=7.4298\right.$, P-value $=0.1148$ ). The detection of malaria infection in relation to diagnostic techniques showed a sensitivity and specificity values of $84.5 \%$ and $98.2 \%$ compared to Microscopy (standard method) which was $100 \%$ for all screened cases.

Conclusion: Symptomatic diagnosis is the most common method of clinical presentation among people of poor resource countries. It should be noted, however, that many other diseases have similar symptoms to malaria and symptomatic diagnosis alone can be misleading and even harmful. Microscopy and RDTs are parts of good clinical practice in the field of medicine and malaria diagnosis, both tools should be employed in the management of malaria treatment and prevention.

\section{Introduction}

Malaria is commonly transmitted by an infected female Anopheles mosquito. The mosquito bite introduces the parasites from the mosquito's saliva into a person's blood [4]. The parasites travel to the liver where they mature and reproduce. Five species of Plasmodium are known to transmit malaria to humans [5]. P. falciparum is the most virulent form of malaria while the other species $P$. vivax, $P$. ovale, and P. malaria

Citation: Mac PA, Asheadzi HF, Gideon A, Thaker P, Airiohuodion P (2019) Prevalence of Plasmodium Falciparum among Nigerians in Abuja and Central States: A Comparative Analysis of Sensitivity and Specificity Using Rapid Diagnostic Test and Microscopy as Tools in Management of Malaria. Int J Trop Dis 1:014

Accepted: January 28, 2019; Published: January 30, 2019

Copyright: (c) 2019 Mac PA, et al. This is an open-access article distributed under the terms of the Creative Commons Attribution License, which permits unrestricted use, distribution, and reproduction in any medium, provided the original author and source are credited. 
generally cause a milder form of malaria $[4,5]$.

In Nigeria, malaria is endemic with co-morbidities such as dengue, chikungunya, Zika, Tuberculosis and HIV/AIDS which accounts for $25 \%$ of infant Mortality and $30 \%$ of childhood mortality [1]. The use of laboratory methods has become necessary because health workers cannot alone identify malaria cases reliably using clinical signs and symptoms alone [2,3]. Conventional light microscopy of a blood smear is the gold standard for the detection of malaria parasites and the established method for the laboratory confirmation of malaria. It is sensitive and can detect densities as low as 5-10 parasites/ $\mu$ l of blood [6]. Microscopy provides information on parasite species ( $P$. falciparum, $P$. vivax, $P$. ovale, and/or $P$. malariae) and their circulating stages (e.g. trophozoites, schizonts, gametocytes), it also aids in the quantification of the parasite densities and assessment of parasitological response to chemotherapy in severe malaria cases.

RDTs are recommended by WHO to enhance diagnosis and management of cases, prevention of complications of delayed treatment, prolonging survival and monitoring of treatment especially in children. Moreover, studies have shown that it is acceptable both to the practicing physicians and the patients [7]. It is timely for case management of malaria and avoids the drawbacks of defective microscope and erratic power supply [7]. It aids in the selective treatment of patients with positive dipstick results and thus slows down the development of drug resistance by preventing drug pressure [8]. However, the proportion of malaria confirmed by laboratory diagnosis in Nigeria is unknown. Diagnosis of malaria is often clinical-based and unreliable, $[2,3,9]$. This could lead to misdiagnosis, over diagnosis, inappropriate treatment and potential development of drug resistance. The use of routine laboratory microscopy to aid clinical diagnosis is minimal. However, the use of malaria rapid diagnostic tests is a new approach and its implementation is restricted to a few facilities in the country due to cost.

This study evaluated prevalence of Plasmodium falciparum using Microscopy and RDT among healthy Nigerians attending Abuja National hospital and Nasarawa State University hospital, Keffias well as the comparative analysis of RDT to Microscopy with respect to sensitivity and specificity.

\section{Methods and Material}

\section{Sampling}

A cross-sectional study made of 403 healthy individuals who came for general medical examinations at National hospital, Abuja and Nasarawa State University hospital, Keffi between April 2017 through July 2017.

\section{Test procedure}

Microscopy method (Gold Standard): A thin film was prepared by placing a drop of blood in the centre of a microscope glass slide and using the corner of a clean slide to spread the blood to cover an area of about 10 $\mathrm{mm}^{2}$ [10]. The slides were labeled using the respective patient numbers assigned at the laboratory. The slides were air dried and stained with $5 \%$ Giemsa's solution for 20 minutes, this was carried out for identification and quantitation of asexual $P$. falciparum species. The slides were then rinsed under mild running tap water and allowed to air-dry. For the Thick film, a small drop of blood was placed at the centre of the grease free slide and spread with the edge of another slide in a repeated coil shaped to a diameter approximately $2 \mathrm{~cm}$. The slides were labeled and left horizontally while drying and were kept well to prevent them from dust and damage. It was stained using $5 \%$ Giemsa stain for 20 minutes and observed microscopically under X100 oil objective lens and result was recorded [10].

Rapid Diagnostic Test (RDT): A rapid lateral flow immuno chromatographic in vitro antigen detection test kit (Care start ${ }^{\mathrm{TM}}$ Access Bio Inc, USA) for detecting malaria $P$. falciparum infection was used to detect malaria HRP2 Pf (Histidine rich protein 2 Plasmodium falciparum) in patient's blood samples according to the manufacturer's instructions. About $5 \mu$ l of blood sample was collected using a micro-pipette provided, the whole blood was added into the "S" well and $60 \mu \mathrm{l}$ assay buffer solution added to the " $A$ " well and result was read after 20 minutes. The diagnostic sensitivity and specificity were determined according to World Health Organization standard, positive and negative predictive value were performed according to Manufacturer's recommendation. Sensitivity refers to the test's ability to correctly detect patients who have malaria and specificity relates to the test's ability to correctly detect patients without a malaria [11].

\section{Determination of sensitivity and specificity:}

Sensitivity (Positive for disease): Sensitivity is the ability of a test to correctly classify an individual as 'diseased'.

Calculation of sensitivity $=\mathrm{TP} / \mathrm{TP}+\mathrm{FN}$

$=\mathrm{TP}$ (true positive)/TP + FN (true positive + false negative)

$=$ probability of being test positive when disease present.

Specificity (Negative for disease): The ability of a test to correctly classify an individual as a disease-free is called the test's specificity.

Specificity - TN/TN + FN

$=\mathrm{TN}$ (true negative) $/ \mathrm{TN}=\mathrm{FN}$ (true negative + false negative)

$=$ Probability of being test negative when disease absent. 
Positive Predictive Value (PPV): It is the percentage of patients with a positive test who actually have the disease. PPV tells us about the number of test positives are true positives; and if these numbers are higher (as close to 100 as possible), then it suggests that this new test is doing as good as 'gold standard'.

$P P V=T P / T P+F P$

PPV $=\mathrm{TP}$ (true positive)/TP + FP (true positive + false positive)

$=$ Probability (patient having disease when test is positive).

Negative Predictive Value (NPV): It is the percentage of patients with a negative test who did not have the disease. NPV tells us how many of test negatives are true negatives; and if this number is higher (should be close to 100), then it suggests that this new test is doing as good as 'gold standard'.

$\mathrm{NPV}=\mathrm{TN} / \mathrm{TN}+\mathrm{FN}$

$=\mathrm{TN}$ (true negative)/FN + TN (false negative + true negative)

= Probability (patient not having disease when test is negative).

\section{Inclusion criteria}

The entry criteria of this study were based on clinician's request for medical examination test for the patients at the National hospital, Abuja and Nasarawa State University hospital, Keffi.

\section{Exclusion criteria}

Patients who had treated malaria three weeks be- fore the research were excluded.

\section{Result}

The prevalence of malaria infection among the 403 subjects was $54.4 \%$ and $44.9 \%$. There was a significant difference between the two methods employed $\left(\chi^{2}=\right.$ $52.171, p<0.001)$. The preponderance of malaria in relation to age was highest 19 (78.9\%; 4.7\%) for ages (above 51 years), those under 20 years demonstrated a pervasiveness of $47(57.4 \% ; 31.9 \%)$ through the two techniques. A prevalence of 25 (64\%; 20\%) was observed in the age group (41.50 years) and to a lesser extend 35 (54.3\%; 28.6\% those years $31-40$ years and 227 (52.3\%; 43.7\%) age 21-30 years (Table 1). There were no significant differences between all age groups $\left(\chi^{2}=7.4298\right.$, P-value $\left.=0.1148\right)$. In this study the prevalence of malaria (Table 2 ) infection among males and females were 180 (54.4\%; 43.3\%) and 223 (47.1\%; $46.2 \%)$ respectively. However, there were no significant differences between the two genders $\left(\chi^{2}=0.44845\right.$, P-value $=0.5031)$. Students in Abuja and Nasarawa metropolis showed a prevalence of 324 (58.3\%; 40.1\%) compared to $25(60 \% ; 32 \%)$ among civil servants and $54(42.6 \% ; 20.4 \%)$ other occupations. There was a significant difference among the different occupations $\left(X^{2}=52.341 ; d f=3, P<0.001\right)$ (Table 3).

The detection of malaria infection in relation to diagnostic techniques showed a sensitivity and specificity values of $84.5 \%$ and $98.2 \%$ (Table 4) compared to Microscopy (standard method) $100 \%$ of all cases. There were no significant differences between Microscopy and RDT sensitivity $\left(\chi^{2}=0.64021, P=0.4236\right)$. A positive and negative predictive values of $95.6 \%$ and $93.1 \%$ were recorded. However, specificity for microscopy and

Table 1: Age related prevalence of malaria parasites among patients.

\begin{tabular}{|l|l|l|l|}
\hline Age (group) & No. examined & No. positive (\%) microscopy & No. positive (\%) RDTs \\
\hline$<20$ & 47 & $27(57.4 \%)$ & $15(31.9 \%)$ \\
\hline $21-30$ & 277 & $145(52.3 \%)$ & $121(43.7 \%)$ \\
\hline $31-40$ & 35 & $19(54.3 \%)$ & $10(28.6 \%)$ \\
\hline $41-50$ & 25 & $16(64 \%)$ & $5(20 \%)$ \\
\hline 51 and above & 19 & $15(78.9 \%)$ & $9(4.7 \%)$ \\
\hline Total & 403 & $222(55.1 \%)$ & $150(37.2 \%)$ \\
\hline
\end{tabular}

$\chi^{2}=7.4298$, P-value $=0.1148$.

Table 2: Gender related prevalence of malaria among patients by light microscopy and RDTs.

\begin{tabular}{|l|l|l|l|}
\hline Gender & No. examined & No. infected (\%) Microscopy & No. infected (\%) RDTs \\
\hline Male & 180 & $98(54.4 \%)$ & $78(43.3 \%)$ \\
\hline Female & 223 & $105(47.1 \%)$ & $103(46.2 \%)$ \\
\hline Total & 403 & $203(54.4 \%)$ & $181(44.9 \%)$ \\
\hline
\end{tabular}

$\chi^{2}=0.44845$, P-value $=0.5031$.

Table 3: Prevalence of malaria infection among occupations.

\begin{tabular}{|l|l|l|l|}
\hline Occupation & No. examined & No. infected (\%) Microscopy & No. infected (\%) RDTs \\
\hline Students & 324 & $189(58.3)$ & $130(40.1 \%)$ \\
\hline Civil servants & 25 & $15(60 \%)$ & $8(32 \%)$ \\
\hline Others & 54 & $23(42.6 \%)$ & $11(20.4 \%)$ \\
\hline Total & 403 & $227(56.3 \%)$ & $149(36.9 \%)$ \\
\hline
\end{tabular}

$\chi^{2}=52.341$, P-value $=0.005$ 
Table 4: Sensitivity and specificity of rapid diagnostic tests in the diagnosis of malaria.

\begin{tabular}{|l|l|l|l|l|l|}
\hline Positive & Negative & Sensitivity (\%) & Specificity (\%) & NPV (\%) & PPV (\%) \\
\hline 114 & 289 & 84.5 & 98.2 & 93.1 & 95.6 \\
\hline
\end{tabular}

PPV: Positive Predictive Value; NPV: Negative Predictive Value.

Table 5: Results of rapid diagnostic using microscopy as the test reference.

\begin{tabular}{|l|l|l|l|}
\hline \multicolumn{2}{|l|}{ Light Microscopy } & Negative & Total \\
\hline RDTs & & & \\
\hline Positive & TP $=109$ & FP $=5$ & 114 \\
\hline Negative & FN $=20$ & TN $=269$ & 289 \\
\hline Total & 129 & 274 & 403 \\
\hline
\end{tabular}

RDT showed no significant difference $\left(\chi^{2}=0.018223, P=\right.$ 0.8926) (Table 5).

\section{Discussion}

There are four main diagnostic methods for malaria. These are symptomatic, microscopic, molecular and antigen testing methods. Symptomatic diagnosis is the most common among people of poorer resource countries.

In this study, we evaluated the prevalence of Plasmodium falciparum employing Microscopy and Rapid Diagnosis Test (RDT) [Histidine Rich Protein-2; HRP-]. Similar studies using different RDTs in Nigeria have been undertaken in various regions $[12,13]$, but such projects employed other RDTS diagnostic kits other than HPR-2 used in the current study. There is no known signature of any study comparing and evaluating the prevalence of malaria in Abuja and Nasarawa among healthy individual employing Microscopy and HPR-2 diagnostic test (RDT). An allover prevalence of (55.1\%; 37.2\%) malaria was observed among all the age groups through Microscopy and RDT, this could be as a result of holo-endemic malaria among Nigerians. Secondly, urbanization and climate conditions have tremendously evolve and enshrine different transmission dynamics of malaria in Abuja and Nasarawa states. This could also be attributed to the fact that the study was conducted in civil servants and student dominated area who are constantly bitten by the female anopheles mosquitoes in their hostels and homes where mosquito impregnated nets are hardly used. Similar studies and literature findings were observed in other parts of Nigeria [14,15]. Subjects of lower age group $(<20)$ had a prevalence of $57.4 \%$ by microscopy and $31.9 \%$ by RDT out of the total 47 subjects screened. Perkin, et al. [2] asserted that, there is slow acquisition of active immunity to malaria; this could be the reason why subjects of lower age groups were susceptible to malaria in this study. Female subjects showed a lower prevalence compared to males in the currents study. The reason for this differences in prevalence between females and males cannot be empirically traced to any reason in particular, it may have occurred by chance. Gilles and Warell [16], reported that there is no scientific evidence that susceptibility to malaria is gender based. The socio-economic status might have also played a role in the observed prevalence common the different occupation. All occupational groups were observed to be predisposed to malaria infections and had a prevalence of (56.3\%; 36.9\%). Onah and Omudu [17] reported that there is no relationship between occupation and prevalence of malaria. This is in contrast to other studies conducted in Akure and ogun staes, $\mathrm{Ni}-$ geria [18-20].

The sensitivity and specificity values for RDT in this study were $84.5 \%$ and $98.2 \%$ compared to (gold standard) microscopy (100\%) technique. Similar findings were reported by Micheal and Florence in Tertiary Healthcare facility in Rivers State, Nigeria [13]. Also, similar results were observed by, Olasehinde, et al. [18] in Ota, ogun- Nigeria using two diagnostic test kits, Bioline SD (HRP-2) and ACON (HRP-2/Aldolase) alongside Microscopy (gold standard) with a sensitivity of $76 \%$ and $100 \%$ respectively. This is however, a total contrast from a similar study conducted at Akure by Obimakinde, et al. [21] which showed a sensitivity of (65\%) RDT compared to Microscopy (71.43\%) and molecular analysis (73.5\%). Sheyin and Bigwan [22] using Carestart HRP 2 reported an sensitivity and specificity values of $78.4 \%$ and $97.6 \%$ in Jos, Nigeria, the study also showed a PPV of $93.3 \%$ and NPV of $80.1 \%$ this is in contrast to our study, where a higher NPV (93.1\%), PPV (95.6\%) and sensitivity of (84.5\%) were observed. This could be attributed to age of the stripes and different manufacturers and methods employed in the study.

Microscopy as gold standard revealed a sensitivity of $100 \%$, the RDT had high Positive predictive value (95.6\%), and this demonstrated that subjects were effectively diagnosed as positive cases of malaria and will be treated and managed accurately. The high Negative predictive value $(93.1 \%)$ means that it was reliable in ruling out malaria. Jang, et al. [23] Care Start HRP-2 reported the sensitivity and specificity for Plasmodium falciparum to be greater than $90 \%$. This study was conducted in different regions of the world, this could have contributed to the variations and differences in results obtained. Similarly a study was carried out in Iran using a RDT, the study detected $71.4 \%$ of Plasmodium vivax while microscopic method detected $(100 \%)$ of all cases [24]. The RDT kit employed in the investigation of the prevalence, sensitivity and specificity of malaria parasite in this study showed an effective and reliable results for detecting Plasmodium falciparum, but not Plasmodium Vivax compare to previous and similar studies conducted in other states in Nigeria and other parts of the world. 
It is therefore imperative to note that this research study is the first in Abuja and Nasarawa state where most malaria infection have been missed or inappropriately diagnosed. With the circulation of arboviral infection among Abuja and the central states, it is appropriate to carry out accurate and effective diagnosis of malaria in order to rule out dengue and other arboviral diseases for better management and treatment outcomes.

\section{Conclusion}

The sensitivity of RDTs at level of parasitaemia and for non-immune population remains a problem compared to stained blood film microscopy. RDTs detection is less sensitive in detecting asymptomatic patient particularly at low parasitaemia [25]. In addition, RDTs often times result in false negative results even at higher parasitemia levels. Consequently, in the event of suspected severe malaria or complex health emergencies, a positive outcome may not exclude malaria. Negative RDTs should however always be microscopically confirmed [26].

Rapid, accurate and accessible detection of malaria parasites is important for prevention, treatment, reduced malaria morbidity, mortality and transmission. Rapid diagnostic tests (RDTS) offer the potential to provide accurate and timely diagnostic to everyone at risk, reaching those previously unable to access good, accurate, and timely diagnosis [26].

The limitations of RDTs include its limited ability to detect 100 parasites/ $\mu \mathrm{l}$ of all plasmodium species and its inability to carry out semi-quantitative measurements to monitor the results of drug treatment. New technologies should be improved on in order to develop new tools that are comparable to Microscopy (gold standard).

The study also highlights the importance of both methods in the prevalence and diagnosis of malaria in Abuja and the central states. Malaria microscopy is still a part of good clinical practices; it should always be a part of malaria case management as it is needed for species identification and confirmation. Although it has a limitations of been laborious, not only is microscopy overwhelming in poor power setting, it is also time consuming and requiring a lot of expertise and training. Many other diseases have symptoms similar to malaria, and symptomatic diagnosis alone may be misleading and even harmful to patient management. Therefore, symptomatic disease should be monitored using both methods. Microscopy is the most widely used tool for the diagnosis of malaria at the peripheral levels and it gives important information about the parasite density and also help improve surveillance in public health practices.

\section{Acknowledgement}

We wish to express our immense gratitude to staff of Nasarawa state University Medical Centre Keffi and
National Hospital and Zankli Medical Centre Abuja, Nigeria.

\section{Statement of Contribution}

All authors contribute equally to this work.

No conflict of interest.

\section{References}

1. Federal Ministry of Health (2006) National framework for monitoring and evaluation of malaria control in Nigeria Abuja, Nigeria, 1-52.

2. Perkins BA, Zucker JR, Otieno J, Jafari HS, Paxton L, et al. (1997) Evaluation of an algorithm for integrated management of childhood illness in an area of Kenya with high malaria transmission. Bull World Health Organ 75: 33-42.

3. Weber MW, Zimmermann U, Van Hensbroek MB, Frenkel $\mathrm{J}$, Palmer A, et al. (1999) Renal involvement in Gambian children with cerebral or mild malaria. Trop Med Int Health 4: 390-394.

4. WHO (2014) World Malaria Report 2014. World Health Organization, Geneva, Switzerland.

5. Caraballo H, King K (2014) Emergency department management of mosquito-borne illness: Malaria, Dengue, and West Nile Virus. Emerg Med Pract 16: 1-23.

6. World Health Organization (1990) Severe and complicated malaria. ( $2^{\text {nd }}$ edn), Transactions of the Royal Society of Tropical Medicine and Hygiene, 84: 1-65.

7. Reyburn $\mathrm{H}$, Mbakilwa $\mathrm{H}$, Mwangi R, Mwerinde $\mathrm{O}$, Olomi $\mathrm{R}$, et al. (2007) Rapid diagnostic tests compared with malaria microscopy for guiding outpatient treatment of febrile illness in Tanzania: Randomised trial. BMJ 334: 403.

8. Rimon MM, Kheng S, Hoyer S, Thach V, Ly S, et al. (2003) Malaria dipsticks beneficial for $\mathrm{IMCl}$ in Cambodia. Trop Med Int Health 8: 536-543.

9. Bell AS, de Roode JC, Sim D, Read AF (2006) Withinhost competition in genetically diverse malaria infections: Parasite virulence and competitive success. Evolution 60: 1358-1371.

10. Cheesbrough M (2000) District Laboratory Practice in tropical countries. ( $2^{\text {nd }}$ edn), University press, Cambridge, 221-245.

11. WHO/LAB (1998) Quality assurance in Haematology. World Health Organization, Geneva.

12. Azikiwe CCA, Ifezulike CC, Siminialayi IM, Amazu LU, Enye JC, et al. (2012) A comparative laboratory diagnosis of malaria: Microscopy versus rapid diagnostic test kits. Asian Pac J Trop Biomed 2: 307-310.

13. Micheal W, Florence N (2016) Validity of presumptive diagnosis of malaria among outpatients in a tertiary healthcare facility in rivers state, Nigeria. $\mathrm{Br} \mathrm{J}$ Med Health Res 3: 52-57.

14. Nwonwu EU, Ibekwe PC, Ugwu JI, Obarezi HC, Nwagbara OC (2009) Prevalence of malaria parasitaemia and malaria related anaemia among pregnant women in Abakaliki, South East Nigeria. Niger J Clin Pract 12: 182-186.

15. Muyiwa K Oyebola, Emmanuel T Idowu , Haddy Nyang, Yetunde A Olukosi , Olubunmi A Otubanjo, et al. (2014) Microsatellite markers reveal low levels of population substructuring of Plasmodium falciparum in southwestern Nigeria. Malar J 13: 493. 
16. Gilles AO, Warell CJ (1993) Estimating the global clinical burden of Plasmodium falciparum in malaria in 2007. PLoS Medicine 7: 6 .

17. Onah IE, Omudu EA (2016) Prevalence of malaria in relation to insecticide treated-nets usage in a rural and urban settlement in Benue State. Nigerian Journal of Parasitology 37.

18. Olasehinde GI, Oyeka UC, Oniha MI, Onile-ere OA, Ayepola OO, et al. (2018) Data set on rapid diagnostic tests (RDTs) and microscopy for diagnosing plasmodium falciparum and plasmodium vivax. Data Brief 20: 503-509.

19. Abah AE, Temple B (2015) Prevalence of malaria parasite among asymptomatic primary school children in Angiama community, Bayelsa State, Nigeria. Tropical Medicine and Surgery 4: 203-207.

20. Kemebradikumo Pondei, Onyaye E Kunle-Olowu, Oliemen Peterside (2012) Patterns of acute febrile illness in children in a tertiary health institution in the Niger Delta Region of Nigeria. J Med Med Sci 3: 734-740.

21. Obimakinde ET, Simon-Oke IA, Osunyemi OS (2018) The effectiveness of microscopy: Rapid diagnostic test and molecular assay in diagnosing malaria. J Parasit Dis Diagn Ther 3: 10-14.
22. Sheyin Z, Bigwan IE (2013) Comparison of care start HRP2 rapid malaria test with light microscopy for guiding patients treatment of fever in Nigerian endemic areas. Journal of Medicine and Medical Sciences 4: 353-356.

23. Jang JW, Cho CH, Han ET, An SS, Lim CS (2013) pLDH level of clinically isolated Plasmodium vivax and detection limit of pLDH based malaria rapid diagnostic test. Malar $\mathrm{J}$ 12: $181-186$

24. Alireza Salimi Khorashad, Masoud Salehi, Bita Roshanravan (2014) The comparison of microscopic method and rapid diagnostic test in detecting Plasmodium species. Int $\mathrm{J}$ Infect 1: 1-3.

25. Elizabeth T Wood, Daryn A Stover, Montgomery Slatkin, Michael W Nachman, Michael F Hammer (2005) The $\beta$-Globin recombinational hotspot reduces the effects of strong selection around $\mathrm{Hbc}$, a recently arisen mutation providing resistance to malaria. Am J Hum Genet 77: 637-642.

26. Leke RF, Rosine R Djokam, Robinson Mbu, Robert J Leke, Josephine Fogako, et al. (1999) Detection of the Plasmodium falciparum antigen Histidine-Rich Protein 2 - in blood of pregnant women: Implication for diagnosing placental malaria. J Clin Microbiol 37: 2992-2996. 